\title{
La métallurgie du silicium solaire
}

Christian Trassy (christian.trassy@grenoble.cnrs.fr)

Science et ingénierie des matériaux et procédés, UMR CNRS 5266, Grenoble

L'industrie photovoltaïque

connaît depuis une

dizaine d'années une

croissance extrêmement

forte, fondée essentiel-

lement sur le silicium

cristallin. Cette croissance

est aujourd'hui limitée

par la disponibilité de

silicium de qualité

solaire. Cette pénurie

rend indispensable

l'émergence d'une filière

d'approvisionnement

spécifique, indépendante

de la filière électronique.

Deux types de solutions

sont aujourd'hui en

compétition :

une filière par voie

gazeuse, dérivée de la

filière électronique,

et un ensemble de

procédés de purification

directe à partir du silicium

métallurgique.
La production de cellules photovoltaïques a dépassé en 2005 1,5 GW-crête ${ }^{1}$. Plus de $93 \%$ de cette production est fournie par des modules au silicium cristallin. Dans les conditions actuelles de production, il faut en moyenne 12 tonnes de silicium pour produire une capacité de 1 MW-crête. C'est plus de 15000 tonnes de silicium de qualité photovoltaïque qui ont été nécessaires en 2005 pour cette production, dépassant la consommation de silicium de l'industrie microélectronique, avec pour résultat une flambée des prix. C'est entre 30000 et 50000 t qui seront nécessaires en 2010. En effet, les rejets de l'industrie microélectronique qui ont pendant longtemps alimenté l'industrie photovoltaique ne suffisent plus, et depuis plusieurs années les " photovoltaïciens " s'approvisionnent directement aux mêmes sources que l'industrie électronique. Le résultat est que le prix du silicium de qualité solaire, qui était de 30 francs par kilogramme dans les années 1997-1998, est passé aujourd'hui à plus de $60 € / \mathrm{kg}$, voire jusqu'à plus de $150 € / \mathrm{kg}$ sur le marché «spot $»^{2}$. Cette situation menace directement la filière photovoltaïque à un moment où la croissance de la demande est particulièrement forte.

\section{La production et la purification du silicium}

Du point de vue du "photovoltaïcien ", la métallurgie du silicium concerne l'ensemble des étapes qui vont conduire des matières premières jusqu'aux plaquettes ${ }^{3}$ brutes, les wafers, à partir desquelles vont être élaborées les cellules. Ces cellules sont ensuite assemblées en modules, unités de production électrique généralement composées de 35 ou 36 cellules dans le cas des modules au silicium cristallin, de façon à fournir une source de tension utilisable dans les systèmes. Cette métallurgie englobe plusieurs étapes : la production de silicium par la réduction de la silice, sa purification et l'élaboration des lingots dans lesquels seront ensuite découpées les plaquettes.

Le silicium est, après l'oxygène, l'élément le plus répandu à la surface du globe :il représente près de $28 \%$ de la croûte terrestre. Cependant il n'existe pas à l'état natif, mais sous forme d'oxydes - la silice - ou de silicates, qui sont des composés extrêmement stables. Le silicium dit «métallurgique " est obtenu par réduction de la silice par le carbone à haute température, dans des fours à arc. La réaction globale s'écrit : $\mathrm{SiO}_{2}+2 \mathrm{C} \rightarrow \mathrm{Si}+2 \mathrm{CO}$

En fait la réaction est plus complexe et on peut schématiquement décomposer le four électrique en deux zones : une zone inférieure chaude, où la température dépasse $1900{ }^{\circ} \mathrm{C}$, et une zone supérieure plus froide. Dans la zone chaude, on a les réactions

$$
\begin{aligned}
& 2 \mathrm{SiO}_{2}(\mathrm{l})+\mathrm{SiC}(\mathrm{s}) \rightarrow 3 \mathrm{SiO}(\mathrm{g})+\mathrm{CO}(\mathrm{g}) \\
& \mathrm{SiO}(\mathrm{g})+\mathrm{SiC}(\mathrm{s}) \rightarrow 2 \mathrm{Si}(\mathrm{l})+\mathrm{CO}(\mathrm{g}) \\
& \text { et dans la zone froide }: \\
& \mathrm{SiO}(\mathrm{g})+2 \mathrm{C}(\mathrm{s}) \rightarrow \mathrm{SiC}(\mathrm{s})+\mathrm{CO}(\mathrm{g}) \\
& 2 \mathrm{SiO}(\mathrm{g}) \rightarrow \mathrm{Si}(\mathrm{l})+\mathrm{SiO}_{2}(\mathrm{~s})
\end{aligned}
$$

On obtient ainsi un silicium de qualité métallurgique dont les principales impuretés sont dues soit au procédé lui-même, SiC et $\mathrm{SiO}_{2}$, soit aux impuretés contenues dans les matières premières ; c'est le cas des impuretés métalliques et des dopants comme le bore et le phosphore. Le taux d'impureté dans ce silicium métallurgique est généralement compris entre 0,5 et $2 \%$. Si, a priori, n'importe quelle silice et n'importe quel réducteur carboné peuvent être utilisés, le fonctionnement fiable et énergétiquement efficace d'un four à silicium impose un choix précis des matières premières et de leur granulométrie ; il est par exemple exclu d'utiliser du sable, fut-il de grande pureté, car on a alors un colmatage du four et une très mauvaise circulation de gaz dans la charge. Les principaux débouchés de ce silicium sont la chimie pour l'industrie des silicones et la métallurgie où il est utilisé comme élément d'alliage. La production annuelle est de l'ordre d'un million de tonnes.

Le silicium de qualité électronique exige une beaucoup plus grande pureté, de l'ordre de $10^{-9}$. Il est préparé par voie gazeuse : du silicium de qualité métallurgique est attaqué à haute température par $\mathrm{HCl}$ pour donner du trichlorosilane, $\mathrm{SiHCl}_{3}$, qui est gazeux. Ce trichlorosilane est purifié par distillation, puis converti en silane, $\mathrm{SiH}_{4}$, lequel par dissociation thermique donne un silicium de grande pureté (voir fig. 1). Les sous-produits sont recyclés. La pureté de ce silicium n'est cependant pas suffisante pour les applications électroniques; une purification complémentaire est apportée par la ségrégation qui intervient lors de la fabrication des lingots dans lesquels sont découpées les plaquettes (wafers) sur lesquelles sont réalisés les composants électroniques. 
Figure 1 : Comparaison des filières d'approvisionnement. À gauche la voie classique, dérivée de la filière électronique (EG-Si), à droite une voie directe à partir de silicium métallurgique amélioré (UMG-Si).

MG-Si : silicium de qualité métallurgique SoG-Si : silicium de qualité solaire photovoltaïque

$P V$-wafers : plaquettes photovoltaïques E-wafers : plaquettes électroniques

\begin{tabular}{|c|c|c|}
\hline & 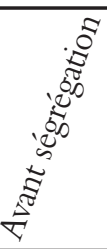 & 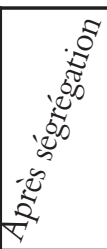 \\
\hline B & 1,6 & 0,9 \\
\hline $\mathrm{P}$ & 4,8 & 2 \\
\hline $\mathrm{Al}$ & 13 & 0,4 \\
\hline $\mathrm{Ca}$ & 9 & $<0,005$ \\
\hline $\mathrm{Cr}$ & 2 & $<0,005$ \\
\hline $\mathrm{Cu}$ & 9 & $<0,01$ \\
\hline $\mathrm{Fe}$ & 30 & $<0,05$ \\
\hline $\mathrm{Ni}$ & $<2$ & $<0,01$ \\
\hline $\mathrm{Ti}$ & 1,5 & $<0,005$ \\
\hline $\mathrm{V}$ & $<2$ & $<0,001$ \\
\hline
\end{tabular}

Tableau 1. Exemple de concentrations d'impuretés avant et après solidification directionnelle (en ppm poids).

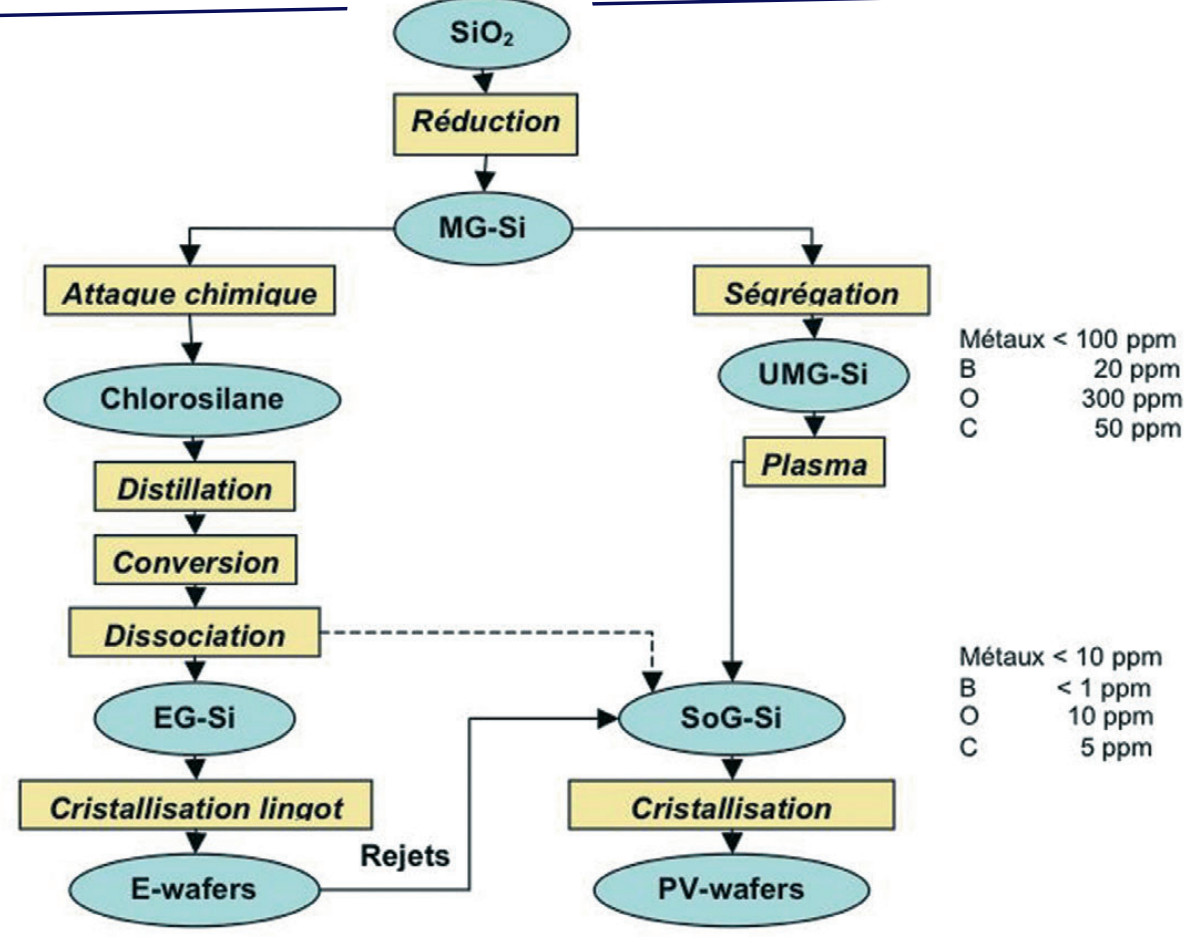

Les applications photovoltaïques sont beaucoup moins exigeantes quant à la pureté du silicium : en général de l'ordre de la ppm (partie par million), soit $10^{-6}$. Pendant longtemps, l'industrie photovoltaïque s'est donc contentée des rejets et des surplus de l'industrie électronique. Cette situation n'est plus tenable au regard de la très forte croissance de la demande : plus de $30 \%$ par an depuis plusieurs années. Le défi auquel doit donc répondre l'industrie photovoltaïque est le développement d'une filière d'approvisionnement qui lui soit propre. Deux types de voies s'ouvrent alors : une voie gazeuse de type électronique simplifiée, et une voie directe à partir du silicium métallurgique. Les recherches dans ce domaine sont très actives, mais aucune de ces voies n'en est encore au stade industriel.

Une voie gazeuse dégradée, principalement par simplification des étapes de distillation, conduira à un silicium relativement coûteux. Elle n'est concevable que dans le cadre d'une filière visant de hauts rendements photovoltaïques, de l'ordre de $20 \%$. Les voies métallurgiques combinent généralement des étapes de ségrégation pour la réduction des impuretés métalliques (voir encadré p. 15) avec d'autres étapes, telles que lavage acide, traitement plasma, canon à électrons ou traitement par laitiers.

Un exemple de combinaison de différentes étapes est donné par le procédé japonais développé initialement par une équipe de Kawasaki Steel [1], dans le cadre du vaste programme photovoltaique japonais du NEDO (agence japonaise de l'environnement). Ce procédé comporte un traitement par canon à électrons pour l'élimination du phosphore, un traitement par plasma réactif d'argon et de vapeur d'eau pour l'élimination du bore, entrecoupés de deux étapes de solidification directionnelle pour la réduction des impuretés métalliques. Ce procédé a permis d'obtenir un silicium de qualité photovoltaïque, mais n'est pas encore viable économiquement. Il est possible d'améliorer l'efficacité de la purification en utilisant un brassage électromagnétique du silicium [2] et en intégrant les différentes étapes, y compris la production des lingots [3].

\section{L'élaboration des lingots}

La production des lingots est une étape importante, car la qualité cristalline du silicium en dépend. Elle constitue en outre une étape significative de purification complémentaire par ségrégation. On distingue les procédés de solidification selon qu'ils conduisent à des lingots monocristallins ou multicristallins.

Parmi les procédés monocristallins, le procédé de la zone flottante (voir encadré p. 15) est celui qui conduit à la plus haute pureté, dans la mesure où le silicium liquide n'est en contact avec aucune paroi ; mais c'est aussi le plus onéreux. Le procédé Czochralsky tire un lingot à partir d'un germe monocristallin plongé dans un bain de silicium liquide ; le creuset en silice est la source d'une pollution en oxygène et le bain liquide s'enrichit progressivement en impuretés, du fait justement de la ségrégation.

Les différents procédés de production de lingots multicristallins (voir fig. 2) reposent tous sur le même principe. Le silicium est fondu dans un creuset en silice, de forme parallélépipédique. À la fin de l'étape de fusion, une perte thermique est créée au fond du creuset et le silicium solidifie progressivement du bas vers le haut. La capacité des creusets peut dépasser $300 \mathrm{~kg}$. 


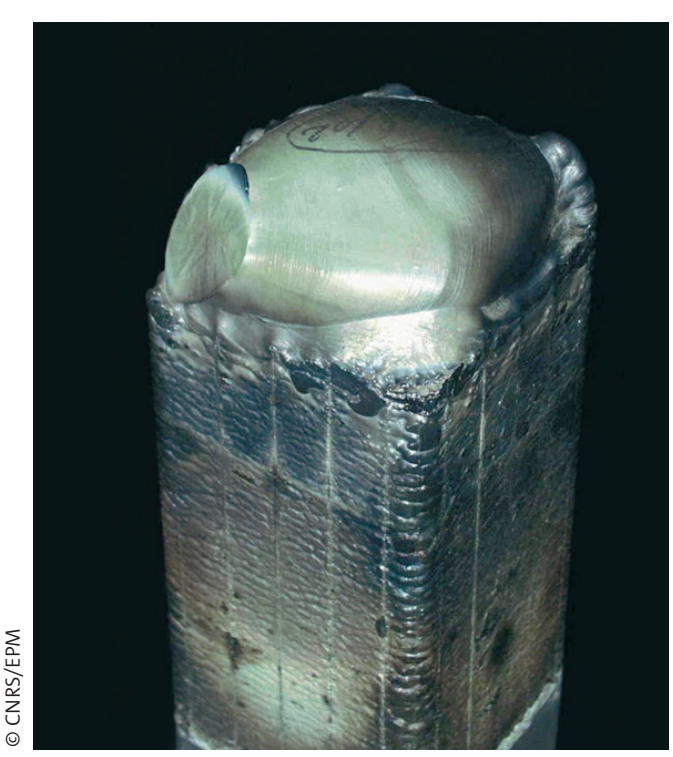

Les grains obtenus peuvent atteindre une longueur de plus de $10 \mathrm{~cm}$ et une section de l'ordre $\mathrm{du} \mathrm{cm}^{2}$. Les rendements obtenus sur silicium multicristallin sont moins élevés que ceux obtenus avec du monocristallin (15 à $16 \%$ contre 17 à $19 \%$, en procédé industriel). Cela tient, entre autres, aux joints de grains où se concentrent les impuretés et qui ont une activité recombinante. Cependant, dans le cas de l'utilisation de silicium de moindre qualité, comme par exemple le silicium métallurgique amélioré, cela pourrait se traduire par un avantage : les joints de grains permettraient de drainer plus efficacement les impuretés métalliques (voir tableau 1). Du point de vue de l'application, il ne faut pas perdre de vue que le critère le plus pertinent n'est pas le rendement de conversion, mais bien le coût du kilowattheure; dans ce coût, le matériau entre aujourd'hui pour une part significative : très grossièrement, dans le coût final d'un module, le wafer entre pour $40 \%$, la fabrication de la cellule pour $20 \%$ et l'assemblage en module pour $40 \%$.
Il se dessine donc deux grandes tendances dans le domaine des cellules à base de silicium massif : une filière hauts rendements qui exige un silicium de grande pureté, et une filière basée sur du silicium de qualité moindre, mais de faible coût. Compte tenu des besoins actuels, ces deux filières, qui toucheront des secteurs différents, ne semblent pas exclusives l'une de l'autre.

\section{Les verrous scientifiques}

L'effet photovoltaïque est aujourd'hui bien compris ; cependant l'application de cet effet à la production d'énergie électrique dépend beaucoup des imperfections du matériau. Les interactions entre défauts et propriétés électriques ont fait et font encore l'objet de beaucoup d'études. Jusqu'à présent, ces études ont principalement porté sur le matériau d'origine électronique. Les effets des impuretés, qui ont fait l'objet de bien des travaux, portent essentiellement sur les interactions binaires, voire ternaires, mais pas au-delà. Très peu d'études concernent le silicium "sale ». Or, dans le cas d'un silicium d'origine métallurgique, c'est près de la moitié de la classification périodique qu'on retrouve à l'état de traces dans le matériau. On retrouve alors une forte influence du procédé de purification sur le type et la forme chimique des impuretés résiduelles ainsi que sur les propriétés du matériau. Les premières cellules réalisées avec du silicium d'origine métallurgique ont montré des rendements de conversion beaucoup plus élevés que ceux que l'on pouvait attendre, si l'on se réfere aux critères de pureté admis jusqu'à aujourd'hui ! Il y a là un champ de recherche quasiment vierge, qu'il sera indispensable de défricher pour pouvoir accéder à des procédés efficaces et à un matériau disponible en abondance.
Figure 2 : Lingot de silicium, de dimensions latérales $10 \times 10 \mathrm{~cm}^{2}$, obtenu par coulée continue électromagnétique.

Le principe de cette technique consiste à fondre le silicium par induction dans un creuset froid métallique sans fond. Le bain fondu est alimenté en continu par des poudres de silicium, tandis que le "pied de lingot " est progressivement descendu. En parvenant hors de la zone d'induction, le silicium se solidifie en un lingot dont la forme est déterminée par la forme du creuset. Ce dernier, qui entre pour une bonne part dans le coût de production du lingot, ne s'use pas et peut être utilisé quasi indéfiniment.

\section{Références}

[1] N. Yuge et al., "Purification of metallurgical silicon up to solar grade", Solar Energy Materials and Solar Cells 34 (1994) 243-250.

\section{[2] C. Alemany, C. Trassy,}

B. Pateyron, K.-I. Li, Y. Delannoy, "Refining of metallurgical-grade silicon by inductive plasma", Solar Energy Materials and Solar Cells 72 (2002) 41-48.

[3] R. Einhaus et al., PHOTOSIL - Simplified Production of Solar Silicon from Metallurgical Silicon, $23^{\text {rd }}$ European Photovoltaic Solar Energy Conference, Dresden, (4-8 sept. 2006).

\section{La purification par solidification directionnelle}

Lorsqu'on solidifie un élément contenant un faible taux d'impuretés, la concentration en impuretés dans la phase solide est généralement beaucoup plus faible que dans la phase liquide. Le coefficient de ségrégation (ou de partage) est défini comme le rapport, à l'équilibre, des concentrations en impuretés entre la phase solide et la phase liquide. Dans le cas du silicium, les coefficients de ségrégation varient de $2 \times 10^{-8}$ pour le tungstène à 0,8 pour le bore ; cela signifie que si le tungstène ségrège très bien, le bore ne ségrège pratiquement pas (c'est pour cela que B est un bon dopant).

Si la solidification est suffisamment lente et contrôlée, c'est-à-dire si on solidifie de façon à ce que phase solide et phase liquide ne se mélangent pas (schéma a), les impuretés sont progressivement rejetées dans le liquide. La dernière fraction solidifiée sera très enrichie en impuretés. Il suffira alors d'éliminer par découpe cette dernière fraction pour obtenir un lingot de silicium très pur. Cette technique convient bien à l'élimination des impuretés à faible coefficient de ségrégation - de façon générale : les impuretés métalliques.

Le procédé de la zone flottante (ou de fusion de zone) est une opération plus complexe, qui consiste à déplacer à vitesse lente et uniforme une zone liquide de faible épaisseur le long d'un barreau solide (schéma b). On transporte ainsi une certaine quantité d'impuretés vers la tête du barreau. Lorsqu'on multiplie le nombre de passages de la zone liquide, on obtient dans les cas favorables un matériau extrêmement pur. 